\title{
Bishop curves and orthogonal trajectories
}

Clark Kimberling and Peter Moses

Clark Kimberling received his Ph.D. in mathematics from the Illinois Institute of Technology, Chicago, in 1970. Since then he has been a member of the mathematics department at the University of Evansville, in Evansville, Indiana.

Peter Moses is an engineer who owns and runs a small company based in the UK. The company specializes in chaplets and rivets composed of mild steel, stainless steel, aluminium, etc. and serves various industries.

\section{Introduction}

In a geometry seminar at the University of Illinois in March 2010, we presented the cubic quadrarc as the intersection of the cylinders $x^{2}+y^{2}=1$ and $x^{2}+z^{2}=1$, and also as the intersection of the sphere $x^{2}+y^{2}+z^{2}=2$ and the cube having vertices $( \pm 1, \pm 1, \pm 1)$. During the discussion, Professor Richard Bishop pointed out that this curve, consisting of four arcs, is only 1-smooth at the joints of arcs. He suggested an intersection of elliptic cylinders, and by varying them we obtain a family of Bishop curves which are everywhere infinitely smooth.

By an "elliptic cylinder" we mean a cylinder whose base is an ellipse. Figures 2 and 3 indicate that for each pair of intersecting elliptic cylinders, one is parallel to the $x$-axis, and the other, to the $y$-axis. In order to tell more about these cylinders (at the end of this section) we begin with the parametric equations given by Professor Bishop. Let $S$ denote the sphere $x^{2}+y^{2}+z^{2}=2$. The $T$-Bishop curve on $S$, for any $T$ in $[-1,1]$, is the union of four arcs, the first given by

$$
x(t)=\sqrt{(1+t)\left(1-T^{2} t\right)}, \quad y(t)=\sqrt{(1-t)\left(1+T^{2} t\right)}, \quad z(t)=\sqrt{2} T t,
$$

Der vorliegende Beitrag ist eine hübsche Ausarbeitung einer Fragestellung aus dem Bereich der Differentialgeometrie von Kurven und Flächen. Die Autoren untersuchen glatte Kurven, die als Durchschnitt von elliptischen Zylindern und einer Sphäre entstehen. Für diese sogenannten Bishop-Kurven werden mit Hilfe der Mercator-Projektion die orthogonalen Trajektorien explizit berechnet. Die Ergebnisse der Arbeit werden durch ansprechende Graphiken illustriert. 


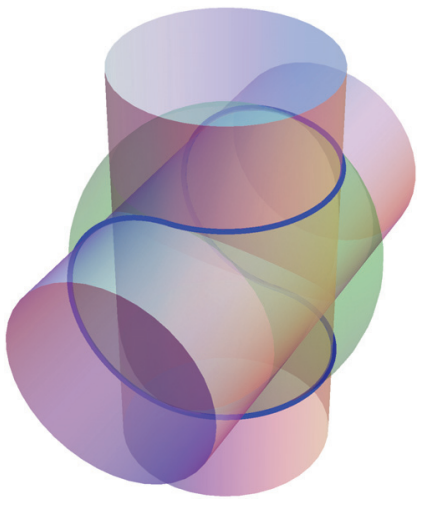

Fig. 1 Cubic quadrarc

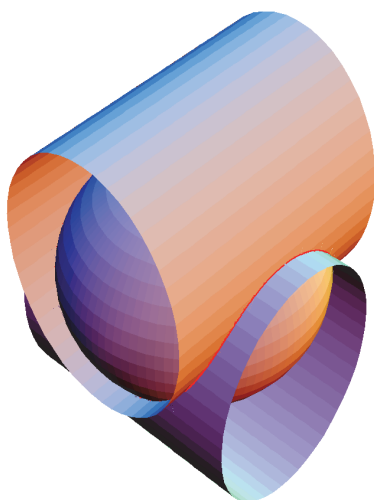

Fig. 2 Bishop curve

where $-1 \leq t \leq 1$. The arcs are then given by

$\operatorname{arc} 1:(x, y, z), \quad \operatorname{arc} 2:(y,-x,-z), \quad \operatorname{arc} 3:(-x,-y, z), \quad \operatorname{arc} 4:(-y, x,-z)$.

Using $U=\sqrt{2-2 T^{2}}$, endpoints and midpoints are shown in Table 1.

\begin{tabular}{|c|c|c|c|}
\hline \multicolumn{4}{|c|}{ Table 1. Eight special points on arcs 1-4 } \\
\hline & $t=-1$ & $t=0$ & $t=1$ \\
\hline \hline arc 1 & $(0, U,-\sqrt{2} T)$ & $(1,1,0)$ & $(U, 0, \sqrt{2} T)$ \\
\hline $\operatorname{arc} 2$ & $(U, 0, \sqrt{2} T)$ & $(1,-1,0)$ & $(0,-U,-\sqrt{2} T)$ \\
\hline $\operatorname{arc} 3$ & $(0,-U,-\sqrt{2} T)$ & $(-1,-1,0)$ & $(-U, 0, \sqrt{2} T)$ \\
\hline $\operatorname{arc} 4$ & $(-U, 0, \sqrt{2} T)$ & $(-1,1,0)$ & $(0, U,-\sqrt{2} T)$ \\
\hline
\end{tabular}

The four points for which $t=0$ lie on the equator, $z=0$. If $T>0$, then arc 1 rises through the equator at $(1,1,0)$, up to $(U, 0, \sqrt{2} T)$, where it meets arc 2 . The curve continues around the sphere, returning to arc 1 .

Ten Bishop curves, obtained by taking $T=0.09,0.19, \ldots, 0.99$, are represented in Figure 3, where they indicate that as $T$ increases, certain angles associated with the $T$-Bishop curves increase, in accord with a one-to-one correspondence with $T$. Let $\alpha$ be the maximalsized angle, from the origin, between the curve and the equator, so that $\alpha$ is the directed angle between the segments $(0,0,0)$-to- $(\sqrt{2}, 0,0)$ and $(0,0,0)$-to- $(U, 0, \sqrt{2} T)$. Let $\beta$ be the directed acute angle that the curve makes wherever it crosses the equator. The correspondence between $T$ and the two angles is then given by

$$
T=\sin \alpha=\tan (\beta / 2) .
$$

It is easy to show that each of the intersecting cylinders has minor axis of length $(1+$ $\left.T^{2}\right) /|T|=2|\csc \beta|$ and major axis of length $2 \sqrt{2}|\csc \beta|$, and that the distance from 


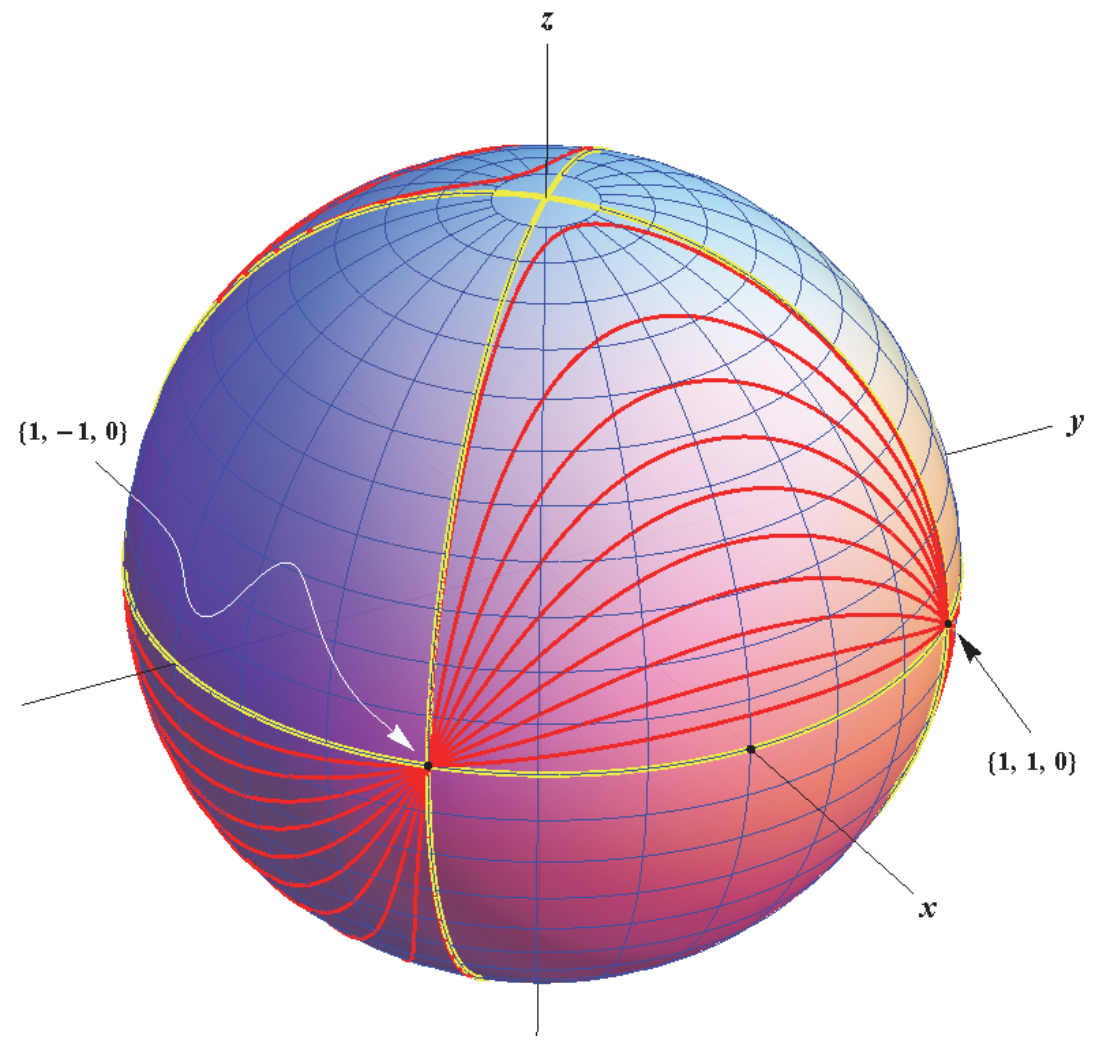

Fig. 3 Ten Bishop curves

either cylindrical axis to the $x y$-plane is

$$
\frac{1-T^{2}}{\sqrt{2} T}=\sqrt{2} \cot \beta=\frac{\cos \alpha \cot \alpha}{\sqrt{2}} .
$$

\section{Another parametrization}

In this section, assume that $T>0$ and project arcs 1 and 2 orthogonally onto the $y z$-plane. The resulting curve is a portion of the ellipse

$$
y^{2}+\frac{z^{2}}{2}+\frac{1-T^{2}}{\sqrt{2} T} z=1 .
$$

Completing the square and putting $y=k_{1} \sin t$ gives $z=\sqrt{2}\left(k_{1} \cos t-k_{2}\right)$, where

$$
k_{1}=\frac{1+T^{2}}{2 T} \quad \text { and } \quad k_{2}=\frac{1-T^{2}}{2 T} .
$$

Then $x=\sqrt{2-y^{2}-z^{2}}$. Using both $(x, y, z)$ and $(-x,-y, z)$, the portion of the Bishop curve thus far accounted for comprises the top half, corresponding to $z \geq 0$, which is to 
say that $-\arccos \left(k_{2} / k_{1}\right) \leq t \leq \arccos \left(k_{2} / k_{1}\right)$. For the bottom half, project arcs 3 and 4 onto the $x z$-plane, and proceed as before. Regarding $(x, y, z)$ as the first of four new arcs that comprise the curve, the final results are as shown here:

$\operatorname{arc} 1^{\prime}:(x, y, z), \quad \operatorname{arc} 2^{\prime}:(-y, x,-z), \operatorname{arc} 3^{\prime}:(-x,-y, z), \operatorname{arc} 4^{\prime}:(y,-x,-z)$.

Using these arcs, we have, in Table 2, the same eight points as in Table 1. Here, however, the joints of neighboring arcs are midpoints in Table 1, and midpoints in Table 2 are joints in Table 1.

\begin{tabular}{|c|c|c|c|}
\hline \multicolumn{4}{|c|}{ Table 2. Eight special points on $\operatorname{arcs} 1^{\prime}-4^{\prime}$} \\
\hline & $t=-\arccos \left(k_{2} / k_{1}\right)$ & $t=0$ & $t=\arccos \left(k_{2} / k_{1}\right)$ \\
\hline \hline $\operatorname{arc} 1^{\prime}$ & $(1,-1,0)$ & $(U, 0, \sqrt{2} T)$ & $(1,1,0)$ \\
\hline $\operatorname{arc} 2^{\prime}$ & $(1,1,0)$ & $(0, U,-\sqrt{2} T)$ & $(-1,1,0)$ \\
\hline $\operatorname{arc} 3^{\prime}$ & $(-1,1,0)$ & $(-U, 0, \sqrt{2} T)$ & $(-1,-1,0)$ \\
\hline $\operatorname{arc} 4^{\prime}$ & $(-1,-1,0)$ & $(0,-U,-\sqrt{2} T)$ & $(1,-1,0)$ \\
\hline
\end{tabular}

The first parametrization shows that for $T<1$, the $T$-Bishop curve is analytically smooth except possibly at the four joints, shown in column 3 of Table 1 . The second parametrization shows that the same curve is analytically smooth at those four points. (Analytically smooth means that at every $u$, there is a neighborhood of $N(u)$ of values $t$ for which there is a parametrization $x(t), y(t), z(t)$ such that each of these has a convergent Maclaurin series; analytic smoothness implies infinite smoothness, in the sense that $x^{(n)}, y^{(n)}, z^{(n)}$ exist and are continuous in $N(u)$.)

\section{Orthogonal trajectories}

Among of the most charming objects in elementary differential equations are orthogonal trajectories - curves in a plane with the remarkable property that wherever one of them meets a curve in a prescribed family, the angle of intersection is $\pi / 2$. In this section, we shall determine families of orthogonal trajectories on a sphere: $x^{2}+y^{2}+z^{2}=R^{2}$, on which longitude $\Phi=\arcsin (z / R)$ and latitude $\Lambda=\arctan y / x$. For the $T$-Bishop curve,

$$
R=\sqrt{2}, \quad \Phi=\arcsin (t T), \quad \Lambda=\arctan \sqrt{\frac{(1-T)\left(1+t T^{2}\right)}{(1+t)\left(1-t T^{2}\right)}} .
$$

Now apply the Mercator angle-preserving mapping to $u v$-plane, using $u=\Lambda$ and $v=$ $\operatorname{arctanh}(\sin \Phi)$. Eliminating $t$ leaves

$$
v=(1 / 2) \operatorname{arcsinh}\left(2 T \cos \frac{2 u}{1-T^{2}}\right)
$$

so that

$$
d v / d u=-2 T \sqrt{1+T^{4}+2 T^{2} \cos (4 u)} \sin (2 u) .
$$




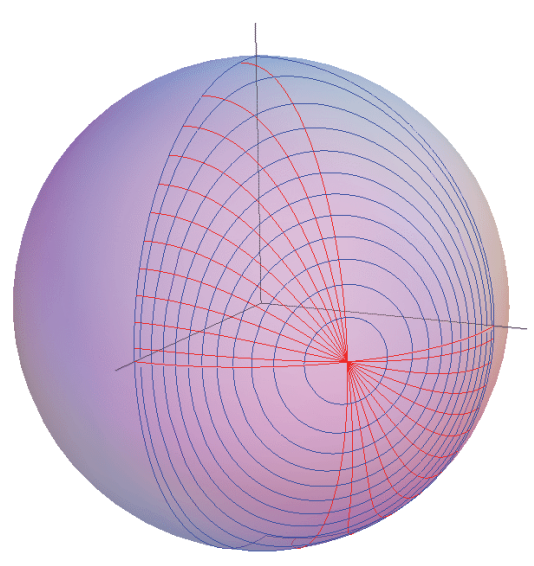

Fig. 4 Bishop curves and orthogonal trajectories

Eliminating $T$ leaves

$$
d v / d u= \pm \tan 2 u \tanh 2 v,
$$

so that the orthogonal trajectories are given by

$$
d v / d u=\mp \cot 2 u \operatorname{coth} 2 v,
$$

with general solution $v= \pm \operatorname{arcsech}(k \csc 2 u)$, where $0 \leq k \leq 1$ and $0 \leq u \leq \pi / 2$.

Next, apply inverse Mercator projection with $\Phi=\arctan (\sinh v)$ and $\Lambda=u$. Writing $t$ for $\Lambda$, we then have parametric equations for the spherical curves which are the orthogonal trajectories of the Bishop curves:

$$
\begin{aligned}
& x(t)=2 \sqrt{\frac{k}{k+\sin 2 t}} \cos t, \\
& y(t)=2 \sqrt{\frac{k}{k+\sin 2 t} \sin t,} \\
& z(t)= \pm \sqrt{\frac{-2 k+2 \sin 2 t}{k+\sin 2 t},}
\end{aligned}
$$

where $0 \leq k \leq 1$ and $(1 / 2) \arcsin k \leq t \leq(1 / 2)(\pi-\arcsin k)$.

An interesting spinoff is yet another parametrization of the $T$-Bishop curve, found as orthogonal trajectories of orthogonal trajectories:

$$
\begin{aligned}
& x(t)=\frac{2 t}{\sqrt{1+\sqrt{1+k^{2}\left(1-2 t^{2}\right)^{2}}}}, \\
& y(t)=\frac{2 \sqrt{1-t^{2}}}{\sqrt{1+\sqrt{1+k^{2}\left(1-2 t^{2}\right)^{2}}}}, \\
& z(t)=\frac{2 k\left(2 t^{2}-1\right)}{\sqrt{1+\sqrt{1+k^{2}\left(1-2 t^{2}\right)^{2}}}},
\end{aligned}
$$




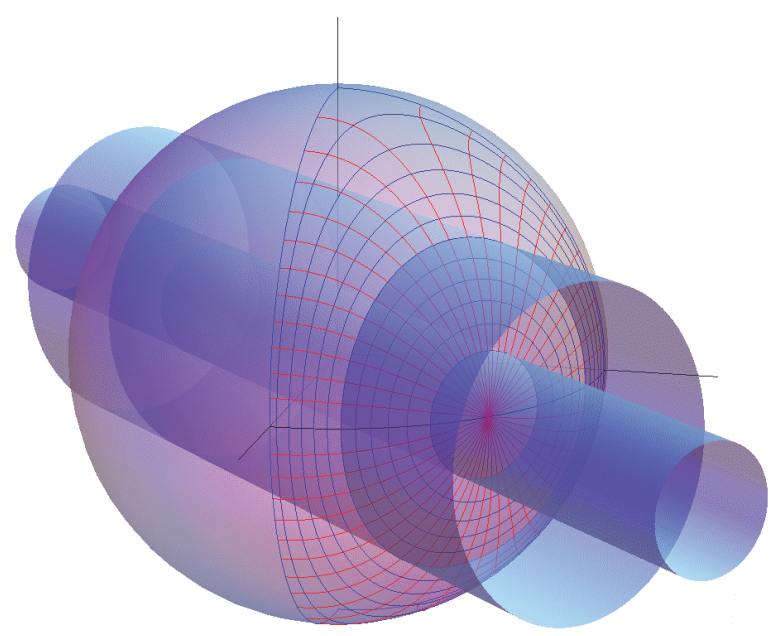

Fig. 5 Elliptic cylinders intersecting sphere in orthogonal trajectories of Bishop curves

where $k=2 T /\left(1-T^{2}\right),-1 \leq T \leq 1,-1 \leq t \leq 1$. This is one of 2 arcs - instead of 4 - so that there are only 2 joints to be examined for infinite smoothness; indeed, by symmetry, we have infinite smoothness at those 2 joints.

Note that, with a small number of exceptions, every point on the sphere lies on exactly one Bishop curve and on exactly one of the orthogonal trajectories.

At an October 2010 session of the aforementioned seminar, Professor John Wetzel, upon viewing Figure 4, suggested that the orthogonal trajectories may be intersections of elliptic cylinders with the sphere. Indeed, the family is given by

$$
\begin{aligned}
& x(\theta)=\sqrt{\frac{1-k}{2}} \cos (\theta-x y), \\
& y(\theta)=\sqrt{\frac{1-k}{2}} \cos (\theta+x y), \\
& z(\theta)=\sqrt{\frac{2-2 k}{1+k}} \sin \theta,
\end{aligned}
$$

where $-\pi<\theta<\pi$. Figure 5 shows two of these cylinders. Be sure to visit the related animation ([2], item 26).

The orthogonal trajectories of Bishop curves are also intersections of hyperbolic cylinders with a sphere. These cylinders are given by

$$
x^{2}+2 x y / k+y^{2}=4
$$

and are typified by the animation ([2], item 28). 


\section{Complementary cylinders}

What others pairs of cylinders intersect in smooth curves on a sphere? We begin with an example: the intersection of a parabolic cylinder and a circular cylinder. Let

$$
x=\sqrt{1+t-t^{2}}, \quad y=\sqrt{1-t^{2}}, \quad z=t,
$$

where $(1-\sqrt{5}) / 2 \leq t \leq 1$, so that $x, y, z$ are all $\geq 0$. Define four arcs by

$\operatorname{arc} 1:(x, y, z), \quad \operatorname{arc} 2:(-x, y, z), \quad \operatorname{arc} 3:(x,-y-z), \quad \operatorname{arc} 4:(-x,-y, z)$.

The curve $G$ consisting of the four arcs has the following properties:

(1) $G$ lies on the sphere $x^{2}+y^{2}+z^{2}=2$;

(2) the orthogonal projection of $G$ onto the $y z$-plane is the part of the parabola given by $z=1-y^{2}$ and $z \geq(1-\sqrt{5}) / 2 ;$ and

(3) the orthogonal projection of $G$ onto the $x z$-plane is the part of the circle given by $x^{2}+(z-1 / 2)^{2}=5 / 4$ and $(1-\sqrt{5}) / 2 \leq z \leq 1 ;$

that is, letting $\varphi=(1+\sqrt{5}) / 2$ be the golden ratio, all of the points of the circle except those satisfying $1<z \leq \varphi$. The curve $G$ is shown in Figure 6 .

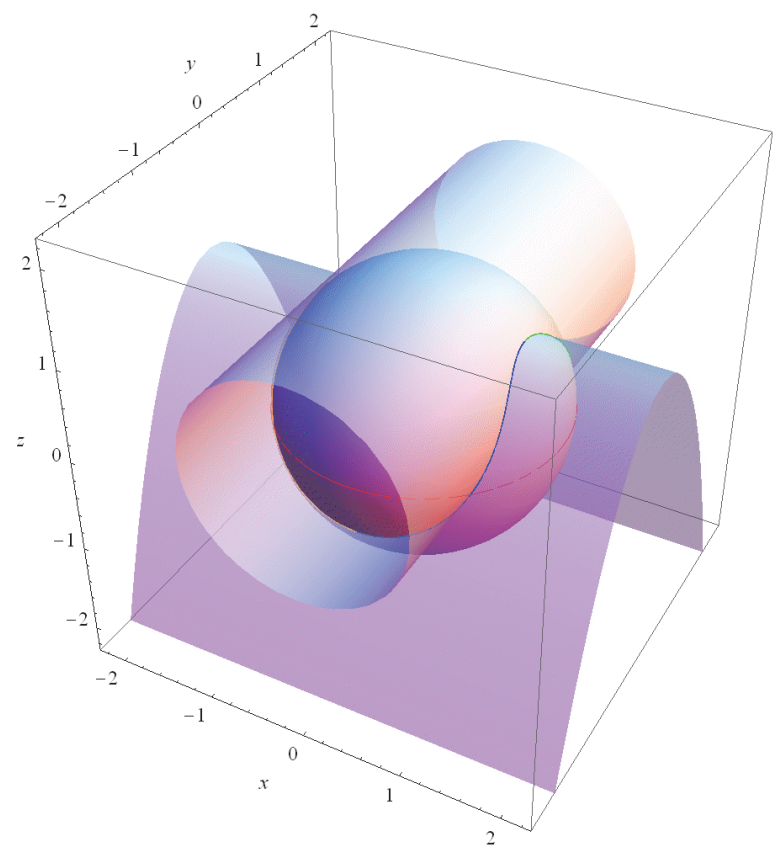

Fig. 6 The curve $G$ as the intersection of the cylinders $x^{2}=1+z-z^{2}$ and $y^{2}=1-z$ 
As the example suggests, one can start with an arbitrary cylinder $\mathcal{C}$ parallel to the $y$-axis and, by intersecting with a sphere $x^{2}+y^{2}+z^{2}=R^{2}$, create a second cylinder, parallel to the $x$-axis. We shall call this second cylinder the complement of $\mathcal{C}$ and denote it by $\mathcal{C}^{\perp}$. If $\mathcal{C}$ is given by $y=f(z)$, then $\mathcal{C}^{\perp}$ is given by

$$
x^{2}=R^{2}-[f(z)]^{2}-z^{2} .
$$

This equation shows that if the $y z$-trace of $\mathcal{C}$ is a conic, then the $x z$-trace of $\mathcal{C}^{\perp}$ is also a conic. Picture 9 in [2] shows intersecting horizontal hyperbolic and elliptic cylinders.

\section{References}

[1] Kimberling, C.; Moses, P.: Gallery of Space Curves Made from Circles. http: / / faculty. evansville.edu/ck6/Gallery/Introduction.html

[2] Kimberling, C.; Moses, P.: Gallery of Bishop Curves and Other Spherical Curves. http: / / faculty. evansville.edu/ck6/GalleryTwo/Introduction.html

Clark Kimberling and Peter Moses

Department of Mathematics

University of Evansville

1800 Lincoln Avenue

Evansville, IN 47722, USA

e-mail: ck6@evansville.edu 